\title{
The relationship between individual phenotype and the division of labour in naked mole-rats: it's complicated
}

\author{
James D Gilbert ${ }^{\text {Corresp., }}{ }^{\text {, }}$, Stephen J Rossiter ${ }^{1}$, Chris G Faulkes ${ }^{\text {Corresp. } 1}$ \\ ${ }^{1}$ School of Biological and Chemical Sciences, Queen Mary University of London, London, United Kingdom \\ Corresponding Authors: James D Gilbert, Chris G Faulkes \\ Email address: j.gilbert@qmul.ac.uk, c.g.faulkes@qmul.ac.uk
}

Background. The naked mole-rat (Heterocephalus glaber) is among the most social mammals on the planet, living in eusocial groups of up to 300 individuals that contain a single reproductive female and up to three reproductive males. A critical aspect of their complex social system is the division of labour that allows non-breeders to form an effective workforce. Age- or weight-based polyethisms are widely cited as explanations for how labour is divided, but evidence in support of these hypotheses has been equivocal.

Methods. To assess the extent to which individual working behaviour is determined by sex, age, weight and social rank, we studied the behaviours of 103 animals from eight captive colonies. We performed focal sampling and ran mixed-effects models to assess which factors explained variation in working behaviour during six ten-minute observation periods per individual.

Results. Contrary to widely-held beliefs, we found that working behaviour did not decrease linearly with weight, although polynomial regressions indicated younger and medium-sized individuals worked most frequently, while high-ranking individuals worked for the shortest periods of time. Working behaviour and its relationship with individual characteristics also varied between colonies.

Conclusions. While age- or size-based polyethisms may have some influence on working behaviour, we argue that other characteristics of the individual and colony are also important. In particular, the interactions of individual, social and environmental factors must be considered in order to understand the emergence and effectiveness of the division of labour that is so critical to many social organisms. 


\section{Revised Manuscript:}

2 The relationship between individual

3 phenotype and the division of labour in

${ }_{4}$ naked mole-rats: it's complicated

5

6 James D. Gilbert*, Stephen J. Rossiter \& Christopher G. Faulkes*

7 School of Biological and Chemical Sciences

8 Queen Mary University of London

9 Mile End Road

10 London E1 4NS

11

12 * corresponding authors - email: j.gilbert@qmul.ac.uk, c.g.faulkes@qmul.ac.uk

13

14

15 


\section{AbSTRACT}

20 Background. The naked mole-rat (Heterocephalus glaber) is among the most social mammals

21 on the planet, living in eusocial groups of up to 300 individuals that contain a single reproductive

22 female and up to three reproductive males. A critical aspect of their complex social system is the

23 division of labour that allows non-breeders to form an effective workforce. Age- or weight-based

24 polyethisms are widely cited as explanations for how labour is divided, but evidence in support

25 of these hypotheses has been equivocal.

26 Methods. To assess the extent to which individual working behaviour is determined by sex, age,

27 weight and social rank, we studied the behaviours of 103 animals from eight captive colonies.

28 We performed focal sampling and ran mixed-effects models to assess which factors explained

29 variation in working behaviour during six ten-minute observation periods per individual.

30 Results. Contrary to widely-held beliefs, we found that working behaviour did not decrease

31 linearly with weight, although polynomial regressions indicated younger and medium-sized

32 individuals worked most frequently, while high-ranking individuals worked for the shortest

33 periods of time. Working behaviour and its relationship with individual characteristics also

34 varied between colonies.

35 Conclusions. While age- or size-based polyethisms may have some influence on working 36 behaviour, we argue that other characteristics of the individual and colony are also important. In

37 particular, the interactions of individual, social and environmental factors must be considered in

38 order to understand the emergence and effectiveness of the division of labour that is so critical to 39 many social organisms. 


\section{INTRODUCTION}

41 The evolution of cooperation is a major driver of sociality and can provide the opportunity for

42 labour to be divided so that individual contributions vary by task or by total effort (Maynard

43 Smith \& Szathmáry, 1999). The benefits of an effective division of labour have been studied in

44 depth in social insects, in which a combination of behavioural flexibility and physical

45

46

47

48

49

50

51

52

53

54

55

56

57

58

59

60

61

62

63

64

65

66

67

68

adaptations meet colony needs (e.g. Wilson 1987; Gordon 1989; Jeanson and Weidenmüller 2014). In contrast, where tasks are divided in social mammal groups, the underlying causes of this division are poorly understood.

Naked mole-rats are widely considered to be eusocial mammals, characterised by an extreme reproductive skew in which $99 \%$ of individuals may never reach reproductive maturity (Jarvis et al., 1994). Breeders specialise in reproduction and, apart from offspring care, contribute little or nothing to general colony work (Jarvis, 1981). Non-breeders are responsible for the critical functions of foraging and nest maintenance, and evidence from the Damaraland mole-rat suggests the presence of additional non-breeders can increase the reproductive output of the colony (Houslay et al., 2020). In regions with low or unpredictable rainfall, the geophytes molerats feed on can be distributed unevenly, while the cost of digging through hard soil is high. Across the African mole-rat family, sociality is thought to mitigate the energetic cost and risks associated with foraging and dispersing in this habitat (Faulkes \& Bennett 2013), although whether cooperative foraging was a cause or consequence of eusociality is unclear (Burda et al 2000). How work is distributed among non-breeders to maximise their output has not been fully explained. Researchers have proposed a number of ways in which tasks could be distributed effectively, often referring to an age- or weight-based polyethism, whereby individuals carry out different types of work according to their age or weight (Jarvis, 1981, 1991; Faulkes et al., 1991; Lacey \& Sherman, 1991).

When Jarvis (1981) first described eusociality in naked mole-rats, she noted that non-breeders were responsible for all the collective work within a colony but did not contribute evenly. Specifically, she observed weight-based polyethisms in which heavier individuals worked less often, seemingly mirroring observations of social insect societies. This observation has grown into the widely-held belief that small individuals exhibit the most working behaviour, typically

Peer] reviewing PDF | (2020:05:49165:1:1:NEW 7 Aug 2020) 
69 encompassing foraging, tunnel maintenance and care of young, while large individuals appear 70 less active, and mainly serve as colony defence.

71 Since this early work, subsequent support for a 'caste-like' system in naked mole-rats has been 72 mixed. For example, Faulkes et al. (1991) examined relationships among weight, sex and various 73 working behaviours, and found evidence for small-workers and large-soldiers. Similarly, Lacey 74 and Sherman (1991) reported that smaller non-breeder do work more, but, due to "considerable 75 scatter" (pp. 322) in behaviours, they concluded that no distinct worker castes are present. Jarvis, 76 O'Riain, and McDaid (1991) also reported mixed results with respect to the relationship between 77 weight and working behaviour. Yet despite these equivocal findings, the concept that naked 78 mole-rat show size-based division of behaviour has become widely adopted as a rule-of-thumb 79 (e.g. Clarke and Faulkes 1997; Jarvis 1981; Jarvis, O’Riain, and McDaid 1991; Lacey and 80 Sherman 1991; Judd and Sherman 1996; Hathaway et al., 2016; Mongillo et al., 2014; Jarvis et 81 al., 1994; Lacey and Sherman 1997). A similar issue exists in social insects, where early reports 82 of polymorphic ant workers with specialised roles has become a widely-held belief that working 83 behaviour is closely linked to body size in general (Gordon, 2016). In reality, in naked mole-rats, 84 while there has been some support for the relationship between body size and aggression towards 85 conspecifics (O’Riain \& Jarvis, 1997; Mooney et al., 2015), empirical evidence for smaller nonbreeders exhibiting more active working behaviour has come from a single study since 1991

87 (Jacobs \& Jarvis, 1996).

88 89 90 91

After almost two decades without further research, a recent analysis has raised further questions regarding earlier findings. Mooney et al. (2015) showed that digging behaviour was not associated with body mass or age, although aggression towards foreign mole-rats was positively correlated with body fat. In light of these results, we performed a large-scale study of the determinants of working behaviour in naked mole-rats. By observing individuals across eight captive colonies, we examined the effects of weight and age on working behaviour. Additionally, we tested for the effect of rank, a characteristic that could influence how much an individual contributes to collective activity but which has not been thoroughly tested (but see Clarke and Faulkes 2001). 


\section{MATERIALS \& METHODS}

99 We selected individuals from eight colonies of captive-bred naked mole-rats at Queen Mary

100 University of London and the study was carried out in accordance with institutional guidelines.

101 They were all captive born descendants of animals that were originally captured in Kenya during

102 the 1980s. Our youngest subjects were four months old during observations as naked mole-rats

103 do not exhibit the full behavioural repertoire until around three months (Jarvis, 1981, 1991).

104 Details of the individuals and colonies used in the study are given in Table 1 (Results, below).

105 Each colony was housed separately in a single room, in a network of acrylic tunnels and boxes.

106 The temperature of the room was maintained between $26-32^{\circ} \mathrm{C}$. Ambient light and noise were

107 constantly recorded and changed unpredictably as people moved around the room and

108 surrounding area. The animals were fed ad libitum every day. The diet generally consists of

109 carrot, sweet potato and butternut squash, although occasionally other fruits and vegetables are

110 provided.

111 We observed all individuals in colonies that contained fewer than 20 in total, and randomly

112 selected 20 individuals from each of the remaining colonies. The observation sequences of

113 colonies and individuals within colonies was also randomly generated. If a colony's breeding

114 female was not selected randomly, she was added to the colony's observation list manually

115 (colonies 11A, 11C and FK100) as a means of comparison in our focal studies. Each individual

116 was observed three times in the morning and three times in the afternoon to avoid confounding

117 variation from daily behaviour patterns. Animals were weighed in each of the three weeks they

118 were observed, and means were calculated and used in the analyses. Animals were identifiable

119 through RFID microchips and were marked twice a week with black marker pens so we could

120 distinguish between them during observations.

121 Females were classified as breeders if they had a perforated vagina during the observation

122 period, they were seen mating, or became pregnant, and classified all other females as non-

123 breeders. External genitalia are generally monomorphic in non-breeding naked mole-rats (Jarvis

124 1991; Peroulakis, Goldman, \& Forger 2002). However, the dark-red vaginal membrane can

125 become prominent in some individuals, which is thought to reflect a partial release from

126 reproductive suppression and may be a sign of incipient reproductive activation (Jarvis 1991). 
127 We recorded which females had prominent vaginal membranes. Males seen mating were 128 recorded as breeders and the rest were classified as non-breeders (Jarvis 1991). While we 129 recorded ano-genital nuzzling when possible, we did not classify animals as breeders if they 130 were observed in these interactions as some ano-genital nuzzling involves non-breeders (Jarvis 131 1991). Historical records of breeding status based on this classification were also used.

132 On entering the animal room, we allowed the colony to settle down from any increased 133 behavioural activity for a period of up to 10 minutes and we minimised movement and noise 134 while observing to avoid disturbing the animals. Behaviours were recorded between 08.00 and 135 18.00. Individuals were observed sequentially in their burrow system for ten minutes each, with 136 each individual observed six times for a total of 60 minutes per individual. All observations were 137 recorded in BORIS (Behavioral Observation Research Interactive Software) version 7.9.7 (Friard $138 \&$ Gamba, 2016).

139 The broad behavioural categories we recorded were as described in the ethogram of naked mole140 rat behaviour: transport of food and nest material, digging and offspring tending (Lacey et al., 141 1991). As it was not possible to distinguish between digging and transporting behaviours, these 142 were classified together as working behaviour. Offspring were only present in one colony during 143 observations and the few instances of tending offspring were not included in the analyses. We 144 recorded reproductive behaviour (Lacey et al., 1991) on an ad hoc basis in order to support 145 hormone analysis in determining reproductive status and to attempt to identify paternity.

146 Reproductive behaviours include copulating, ano-genital nuzzling, pregnancies and births.

147 We tested for an effect of dominance rank on working behaviour and aggression by establishing 148 the dominance hierarchy within each colony using passing behaviour, which is a reliable 149 indicator of dominance hierarchies in naked mole-rats (Clarke \& Faulkes, 1997). We recorded 150 which individuals passed over the top of other individuals during face-to-face encounters in 151 tunnels. Interactions not thought to indicate rank include tail-to-face encounters, passing in 152 chambers or corners of tunnels, when one individual digs throughout the encounter, and when 153 individuals do not pass directly over the top of one another.

154 We used the Elo Rating system to calculate individual dominance ranks within colonies (R 155 package EloRating, Neumann \& Kulik, 2020). The Elo Rating method has several advantages 156 over matrix-based methods such as its ability to calculate ranks within small groups and account 
157 for the loss of individuals during observations. After calculating each individual rank, we scaled

158 each rank to account for variation in group size by dividing by the number of individuals

159 observed in the respective colony. Lower rank values (those towards zero) represent more

160 dominant animals. We calculated the steepness of each colony's dominance hierarchy using the

161 steepness function from the EloRating package, which is based on David's Scores (de Vries,

162 Stevens \& Vervaecke, 2006).

163 10-minute observation periods that contained no working behaviour were assigned a value of

164 zero. To investigate which factors predicted whether an individual showed any working

165 behaviour, we conducted logistic mixed-effect models, using the R package lme4 (Bates et al.,

166 2020, p. 4). For individuals that showed working behaviour in a given session, we determined

167 the factors that predicted variation in the duration of working behaviour using linear mixed-

168 effect models, also in lme4. For a discussion of "two-part" models, see (Duan et al., 1983; Min \&

169 Agresti, 2002). We checked the residuals of the logistic models for uniformity, dispersion, zero-

170 inflation and the presence of outliers using the DHARMa R package (Hartig, 2020). For the

171 linear models, we confirmed the residuals were normally distributed and had similar variances.

172 In both our logistic and linear models, we first constructed null models in which we included

173 individual and colony fitted as random effects, with individual nested within-colony. The

174 response variable was working behaviour per observation session. For each null model, we then

175 constructed a set of separate models, each containing one of the individual characteristics as a

176 fixed effect: sex, age, weight and rank. Due to the correlations between age, weight and rank

177 (older individuals tend to be bigger and of higher rank, Schieffelin and Sherman 1995; Clarke

178 and Faulkes 1997; O'Riain and Jarvis 1998;) we did not create models that contained more than

179 one fixed effect. As relationships between work and age, weight and rank could be non-linear,

180 we also created polynomial linear regressions that included the individual characteristic-squared

181 and -cubed. We compared the performance of the models as described below and report the best

182 performing for each characteristic.

183 Akaike Information Criterion (AIC) values estimate how well a model approximates the

184 unknown reality relative to other models; smaller AIC values indicate better models (Burnham \& 185 Anderson, 2002). Second-order AICs (AICcs) were generated using the aictab function from the 186 AICcmodavg package (Mazerolle \& Linden, 2019) for each model and compared to see whether 
187 the inclusion of an individual characteristic reduced the AICc. The relative likelihood of a model 188 given the data is calculated by $\exp (-(1 / 2) \triangle A I C c)$, where $\triangle \mathrm{AICc}$ is the difference in AICc between 189 two models (Burnham, Anderson \& Huyvaert, 2011). The ratios of model likelihoods can be 190 used to calculate an evidence ratio, which indicates the extent to which the data support one 191 model over another (Burnham, Anderson \& Huyvaert, 2011). We report the AICc values, $192 \Delta$ AICcs and likelihoods relative to the null model for each alternative model. Along with AIC 193 values as indicators of relative model performance, we report Nakagawa's $\mathrm{R}^{2}$ for each model to 194 estimate how much variation in our working behaviour data is explained by the independent 195 variables (Nakagawa \& Schielzeth, 2013; Nakagawa, Johnson \& Schielzeth, 2017), calculated 196 using the performance $\mathrm{R}$ package (Lüdecke et al., 2019).

197 All statistical analyses were carried out in R Studio Version 3.6.0 (R Core Team, 2014) and 198 figures were made using the ggplot2 package (Wickham, 2016). 


\section{RESULTS}

200 We focally sampled a total of 133 Individuals. After excluding breeders, females with red

201 vaginal membranes and individuals with missing sex, age, weight or rank data, a total of 103

202 individuals were used in the analyses. Each individual was observed six times, giving a total of

203618 observation periods. Working behaviour was observed in 274 (44\%) observation periods and

204 no working behaviour was observed in 344 periods (56\%). The average duration of working

205 behaviour observed per ten-minute observation was 166.6 seconds, accounting for $19.4 \%$ of the

206 total observation time. This is comparable to the $23.8 \%$ of time spent working by humans during

207 a 40-hour week. Working behaviour per individual is broken down by sex, age, weight and rank

208 in Figure 1, Figure 2, Table 1 and Table 2, below.

209 Table 1. Summary Statistics

210 Table 2. Working behaviour by Sex 
211 Figure 1A-C. Presence of working behaviour by age, weight and rank of the individual

212 being observed.

213 Figure 2A-C. Duration of working behaviour by age, weight and rank of the individual 214 being observed.

215 To assess which characteristics predicted whether worker behaviour was observed or not, we

216 compared each of our logistic regression models with a single fixed effect to the null model and

217 assessed model fit. $\triangle \mathrm{AIC} c s$ showed that sex, weight and rank did improve model fit $(\Delta \mathrm{AIC} c s$,

218 versus null model: Sex $=+1.48$, Weight $=+0.79$, Rank $=-0.06$; model outputs in Tables 3, SI5).

219 Effect sizes and standard errors support the inference that these characteristics do not explain

220 variation in whether an individual was observed working. In contrast, adding age was associated

221 with a reduction in AICc value of 7.24 and the likelihood of this model was approximately 37

222 times higher than that of the null model. The model coefficient suggests individuals were less

223 likely to be observed working as age increased and this result was significant at alpha=0.05.

224 While including weight did not improve the performance of the model (SI5), including weight-

225 cubed reduced the AICc by 3.89 in a model that had a likelihood 7 times higher than the null

226 model. Nakagawa's $\mathrm{R}^{2}$ estimates suggest all models had limited explanatory power (conditional

$227 \mathrm{R}^{2}$ between 0.202 and 0.230 ), including those that performed better according to AICc, and more

228 variation was explained by the colony and individual random effects than by any of the fixed

229 effects (Table 3).

230

231

232

233

234

235

236

237

238

239

240

Table 3. Results of logistic mixed-effects models predicting whether working behaviour was observed or not.

To assess which characteristics predicted the duration of work observed, we compared each of our linear models with a single fixed effect to the null model and assessed model fit. Linear models excluded observation periods during which no working behaviour was recorded. $\triangle \mathrm{AICc}$ values suggest adding sex, age, weight or rank as predictors did not improve the null model, which included only colony as a random effect $(\triangle \mathrm{AICcs}$, versus null model: $\mathrm{Sex}=+2.04$, Age $=$ +2.00 , Weight $=+1.28$, Rank $=+0.56$; model outputs in Tables 4, SI5). Effect sizes and standard errors support the inference that these characteristics do not predict variation in the amount of time individuals were observed working. While including rank did not improve the performance of the model (SI5), including rank-squared reduced the AICc by 7.21 in a model that had a 
241 likelihood 37 times higher than the null model. Figure $2 \mathrm{C}$ suggests higher ranking individuals

242 may work for shorter periods, although this effect appears to plateau and may even reverse

243 among mid- and low-ranking animals. Nakagawa's $\mathrm{R}^{2}$ estimates suggest all models had limited

244 explanatory power (conditional $\mathrm{R}^{2}$ between 0.169 and 0.182 ), including those that performed

245 better according to $\mathrm{AICc}$, and more variation was explained by the colony and individual random

246 effects than by any of the fixed effects (Table 4).

247 Table 4. Results of generalised linear mixed-effects models predicting the duration of 248 observed working behaviour.

249

250

In summary, age did predict whether an individual worked or not, but was not associated with

251 the duration over which an individual worked. None of the other variables (sex, weight and rank)

252 predicted either whether an individual was recorded working or not, or the duration of working

253 behaviour. Weight-cubed also predicted whether an individual was observed working, while

254 rank-squared predicted the duration of working behaviour. None of the models had high

255 explanatory power and the majority of the variation in working behaviour was unexplained.

256 Plots of the duration of working behaviour and the relationships between working behaviour and

257 individual characteristics within-colonies demonstrate the between-colony variation in our data

258 (Figure 3, Figure 4, Figure 5). Each colony's dominance hierarchy was assessed for how steep it 259 was and assigned a value between 0 and 1, with 1 being the steepest. All steepness estimates 260 were below 0.2 .

261 Figure 3. The mean duration of working behaviour per observation period for each colony.

262 Figure 4A-C. Presence of working behaviour by age, weight and rank of the individual 263 being observed for each colony.

264 Figure 5A-C. Duration of working behaviour by age (A), weight (B) and rank (C) of the 265 individual being observed for each colony. 


\section{DISCUSSION}

268 Previous studies indicate that some naked mole-rats spend more time working than others

269 (Jarvis, 1981; Lacey \& Sherman, 1991) and that differences between individuals may be stable

270 for long periods of time (Mooney et al., 2015). Our analyses of 618 observation periods,

271 encompassing 103 non-breeders from eight colonies, indicated that the probability of being

272 observed working was explained by age and weight-cubed but not by sex, weight or rank.

273 Initially, work frequency seems to increase with age and weight, until intermediate weights and

274 around the age of two, after which the frequency of work plateaus and may even decrease

275 (Figure 1). The assignment of different roles to different age or weight classes distributes work

276 across a colony without the need for costly cognitive evaluations of colony needs and worker

277 availability based on patchy information (Robinson, 1992). Indeed, the presence of overlapping

278 generations of offspring is considered a key criterion for eusociality (Crespi \& Yanega, 1995)

279 and different age and weight cohorts will almost always be present within eusocial groups.

280 Despite the effect of age on probability of working, when we excluded periods in which no

281 working behaviour was recorded, we found that only rank-squared predicted the amount of time

282 spent working during the observation period. Figure 2C suggests low- and mid-ranking

283 individuals worked for longer periods. Work duration was shorter among high-ranking, more

284 dominant individuals.

285 In the absence of clear linear predictors, we suggest that age- or size-based polyethisms may 286 depend on other conditions, such that colony members alter their working behaviour in response

287 to the complex interaction of individual- and group-level pressures. Indeed, Mooney et al. (2015)

288 found that individuals changed their behaviours to compensate for the removal of their colony

289 mates, indicating a level of flexibility in response to the needs of the group. Moreover, a

290 relationship between age and behaviour was only evident when frequent-workers were removed,

291 for which younger individuals compensated by increasing their work rate (Mooney et al., 2015).

292 On the other hand, in the eusocial Damaraland mole-rat (Fukomys damarensis), age does seem to

293 play a key role in cooperative behaviour (Zöttl et al., 2016; Thorley et al., 2018). Both studies of

294 Damaraland mole-rats found helping behaviours increased until the age of one, after which point

295 there was either a plateau (Zöttl et al., 2016) or reduction (Thorley et al., 2018) in helping

296 behaviour. The similarity of helping behaviour in Damaraland and naked mole-rats is interesting, 
297 and implies convergent evolution of these patterns given that sociality is thought to have evolved 298 separately in the two species (Faulkes \& Bennett 2013).

299 Taken together, our findings imply that older individuals perform fewer bouts of working, 300 although these bouts do not differ in duration compared to those of other individuals. In 301 mammals, older individuals tend to be less active (Ingram 2000, Marck et al., 2017) and our 302 results may reflect this general mammalian trend. There is also evidence activity decreases after 303 the of age three in the Ansell's mole-rat (Schielke, Begall \& Burda, 2012). The relatively small 304 number of working bouts seen in older mole-rats might also relate to them having other roles, 305 which were not recorded here. For example, while we did not analyse aggressive or defensive 306 behaviours, it is possible that older individuals, who also tend to be larger (O'Riain \& Jarvis, 307 1998), spend more time defending the colony (Mooney et al., 2015). Older individuals may also 308 invest less in working behaviour in order to maintain energy reserves for future challenges for 309 dominance within the colony, particularly given that these animals are more likely to succeed in 310 the event that a breeder dies or is removed (Reeve, 1992; Clarke \& Faulkes, 1997, 1998).

311 We found no evidence that work decreases linearly with body size, but work frequency increases 312 with size until intermediate body weights are reached. After this, work frequency plateaus and 313 may decrease. However, we are cautious in our interpretation of this result due to the relatively 314 sparse data from animals of small and large body weight, and the tendency of polynomial models 315 to overfit data (Lever, Krzywinski \& Altman, 2016).

316 Across the African mole-rats, the link between age and size on working behaviour is still far 317 from clear. For example, in naked mole-rats, Mooney et al. (2015) also reported no relationship 318 between body size and working behaviour, although larger individuals were more aggressive 319 towards conspecifics (O'Riain \& Jarvis, 1997). On the other hand, body size does seem to be a 320 good predictor of space-use in the Ansell's mole-rat (Skliba et al., 2016). In contrast, a reported 321 association between size and working behaviour in the social Micklem's mole-rat (Fukomys 322 micklemi) was attributed to an underlying age-based polyethism (Van Daele et al., 2019), while a 323 similar association in the Damaraland mole-rat may relate to growth rates (Zöttl et al., 2016). An 324 early report on Damaraland mole-rats found that heavier individuals worked more (Gaylard, 325 Harrison \& Bennett, 1998). In this respect, it is interesting to note that naked mole-rats exhibit 326 unusual growth patterns; growth rates vary between litters and individuals can gain size rapidly 
327 in response to social changes, even after extended periods of constant size (O'Riain \& Jarvis,

328 1998). This decoupling of size and age may further complicate the relationship with behaviour.

329 We found no linear effect of social rank on working behaviour, but did find an effect of rank-

330 squared. Naked mole-rat groups have strict dominance hierarchies (Clarke \& Faulkes, 1997) and

331 periods with high rates of aggression and fighting during which individuals are often killed

332 (Clarke \& Faulkes, 1997, 2001; Medger et al., 2019). High-ranking naked mole-rats are more

333 likely to become breeders if the previous breeders are removed (Clarke and Faulkes, 1997,

334 1998), and may reduce energy spent on working behaviour in order to prepare for future

335 dominance challenges. Alternatively, high-ranking individuals tend to be older and heavier

336 (Clarke and Faulkes, 1997, 1998) and may contribute less to general working behaviour but more

337 to colony defence, as has been reported elsewhere (O'Riain \& Jarvis, 1997; Mooney et al.,

338 2015). More data are need before we can be certain about the distribution of work among

339 heavier, older and higher-ranking individuals. In our study, we recorded an average of four

340 interactions per individual, whereas the Elo-Rating method for assigning rank performs better

341 when there are around ten interactions per individual (Sánchez-Tójar, Schroeder \& Farine,

342 2018). Thus, given the large confidence intervals around estimates of rank, we would like further

343 evidence to confirm the impact of rank, which might be more evident over longer observational

344 periods.

345 Our observations reveal considerable variation in the total amount of working and the

346 relationships between work and individual characteristics within each colony (Figures 3, 4, 5).

347 With data from eight colonies, we did not have the power to test colony-level hypotheses,

348 however, individual behaviours may be affected by a number of colony-level factors, such as

349 food availability (Reeve, 1992), worker availability (Mooney et al., 2015) or the age-structure of

350 the colony (Gaylard, Harrison \& Bennett, 1998, Skliba et al., 2016). Many published results from

351 naked mole-rat behaviours have come from studies of one or a few colonies and were thus

352 unable to account for colony-level variation. In this study, more variation in working behaviour

353 was explained by individual and colony variables than by any individual characteristics. Our

354 findings indicate that future research should increase the number of colonies used and

355 statistically control for colony-level effects wherever possible. 
356 This study looked at the factors that could predict whether an individual works and the variation

357 in the amount of time individuals spend working. Labour could also be divided by task. Task

358 specialisation has been observed in some social insect species (Charbonneau \& Dornhaus, 2015)

359 and, although it could be an important benefit to sociality in mammals, it has rarely been

360 recorded (Stander, 1992; Gazda et al., 2005; Hurtado, Fénéron \& Gouat, 2013; Gazda, 2016).

361 Mooney et al. (2015) found that the amount of time spent on different tasks was stable in naked

362 mole-rats, although Thorley et al. (2018) found no evidence for task specialisation in the eusocial

363 Damaraland mole-rat. We could not test worker specialisation due to the lack of pup tending

364 observed. Although some authors have shown that repeated exposure to a task increases

365 efficiency in insects (Langridge, Sendova-Franks \& Franks, 2008), this is not always the case

366 (Dornhaus, 2008; Santoro, Hartley \& Lester, 2019). Future research could use experimental tasks

367 to explore the presence of specialisation in naked mole-rats and determine whether it increases

368 individual or colony efficiency. Assessing worker, pup tending and defence behaviours like

369 Mooney et al. (2015) would be the best way to establish whether task specialisation exists, as has

370 been suggested in studies of other mole-rats (Van Daele et al., 2019).

\section{CONCLUSION}

372 Gordon (2016) suggested that the term 'division of labour', implying internally-driven choices, is

373 too rigid, and instead terms relating to individual behaviours should emphasise the influences of

374 external conditions and social interactions. Further, we agree that workers should not be

375 classified according to discrete 'castes' in mole-rats unless clear supporting evidence is reported

376 (Skliba et al., 2016). Polyethisms based on individual characteristics could be the start of an

377 effective division of labour but the relationship appears to be complicated and variable. The

378 outstanding questions are the extent to which polyethisms can be fine-tuned and the mechanisms

379 that facilitate these changes. To date, the causes of within-individual variation are still largely

380 unstudied in social mammals and insects (Jeanson, 2019). Given our results, combined with

381 those of Mooney et al. (2015) and the mixed findings of earlier studies (Faulkes et al., 1991;

382 Jarvis, 1991; Lacey \& Sherman, 1991), future research should focus on the interaction between

383 internal, social and environmental influences on working behaviour, rather than attributing such 
384 strong influence to internal factors. As Gordon (2016) argues, interchangeability and the absence 385 of fixed specialisation are exactly what make collective behaviour flexible and adaptive.

386 


\section{REFERENCES}

388

389 Bates D, Maechler M, Bolker B, Walker S, Christensen RHB, Singmann H, Dai B, Scheipl F,

390 Grothendieck G, Green P, Fox J. 2020. Ime4: Linear Mixed-Effects Models using

391 "Eigen” and S4.

392

393

394

395

396

397

398

399

400

401

402

403

404

405

406

407

408

409

Burda H, Honeycutt RL, Begall S, Locker-Grütjen O, Scharff A. 2000. Are naked and common mole-rats eusocial and if so, why?. Behavioral Ecology and Sociobiology 47:293-303. DOI: $10.1007 / \mathrm{s} 002650050669$

Burnham KP, Anderson DR. 2002. Model Selection and Multimodel Inference: A Practical Information-Theoretic Approach. New York: Springer-Verlag. DOI: 10.1007/b97636.

Burnham KP, Anderson DR, Huyvaert KP. 2011. AIC model selection and multimodel inference in behavioral ecology: some background, observations, and comparisons. Behavioral Ecology and Sociobiology 65:23-35. DOI: 10.1007/s00265-010-1029-6.

Charbonneau D, Dornhaus A. 2015. Workers 'specialized' on inactivity: Behavioral consistency of inactive workers and their role in task allocation. Behavioral Ecology and Sociobiology 69:1459-1472. DOI: 10.1007/s00265-015-1958-1.

Clarke FM, Faulkes CG. 1997. Dominance and queen succession in captive colonies of the eusocial naked mole-rat, Heterocephalus glaber. Proceedings of the Royal Society B: Biological Sciences 264:993-1000. DOI: 10.1098/rspb.1997.0137.

Clarke FM, Faulkes CG. 1998. Hormonal and behavioural correlates of male dominance and reproductive status in captive colonies of the naked mole-rat, Heterocephalus glaber. Proceedings of the Royal Society B: Biological Sciences 265:1391-1399. DOI: 10.1098/rspb.1998.0447. 
410 Clarke FM, Faulkes CG. 2001. Intracolony aggression in the eusocial naked mole-rat,

411 Heterocephalus glaber. Animal Behaviour 61:311-324. DOI: 10.1006/anbe.2000.1573.

412 Crespi BJ, Yanega D. 1995. The definition of eusociality. Behavioral Ecology 6:109-115.

413 Dornhaus A. 2008. Specialization Does Not Predict Individual Efficiency in an Ant. PLOS

414 Biology 6:e285. DOI: 10.1371/journal.pbio.0060285.

415 Duan N, Manning WG, Morris CN, Newhouse JP. 1983. A Comparison of Alternative Models 416 for the Demand for Medical Care. Journal of Business \& Economic Statistics 1:115-126.

418

419

420

421

422 423

424

425

426

427

428

429

430

431 DOI: $10.2307 / 1391852$.

Faulkes CG, Abbott DH, Liddell CE, George LM, Jarvis JUM. 1991. Hormonal and behavioral aspects of reproductive suppression in female naked mole-rats. In: Sherman PW, Jarvis JUM, Alexander RD eds. The Biology of the Naked Mole-Rat. NJ: Princeton University Press, 426-445.

Faulkes CG, Bennett NC. 2013. Plasticity and constraints on social evolution in African molerats: ultimate and proximate factors. Philosophical Transactions of the Royal Society B: Biological Sciences. 368: 20120347. DOI: 10.1098/rstb.2012.0347

Friard O, Gamba M. 2016. BORIS: a free, versatile open-source event-logging software for video/audio coding and live observations. Methods in Ecology and Evolution 7:13251330. DOI: 10.1111/2041-210X.12584.

Gaylard A, Harrison Y, Bennett NC. 1998. Temporal changes in the social structure of a captive colony of the Damaraland mole-rat, Cryptomys damarensis: the relationship of sex and age to dominance and burrow-maintenance activity. Journal of Zoology 244:313-321. DOI: 10.1111/j.1469-7998.1998.tb00035.x. 
432 Gazda SK. 2016. Driver-barrier feeding behavior in bottlenose dolphins (Tursiops truncatus):

433 New insights from a longitudinal study. Marine Mammal Science 32:1152-1160. DOI:

434 10.1111/mms.12314.

435

436

437

438

439

440

441

442

443

444

445

446

447

448

449

450

451

452 group-hunting bottlenose dolphins (Tursiops truncatus) off Cedar Key, Florida. Proceedings of the Royal Society B: Biological Sciences 272:135-140. DOI: 10.1098/rspb.2004.2937.

Gordon DM. 1989. Dynamics of task switching in harvester ants. Animal Behaviour 38:194-204. DOI: $10.1016 / \mathrm{S} 0003-3472(89) 80082-\mathrm{X}$

Gordon DM. 2016. From division of labor to the collective behavior of social insects. Behavioral Ecology and Sociobiology 70:1101-1108. DOI: 10.1007/s00265-015-2045-3.

Hartig F. 2020. DHARMa: Residual Diagnostics for Hierarchical (Multi-Level / Mixed) Regression Models.

Hathaway GA, Faykoo-Martinez M, Peragine DE, Mooney SJ, Holmes MM. 2016. Subcaste differences in neural activation suggest a prosocial role for oxytocin in eusocial naked mole-rats. Hormones and Behavior 79:1-7. DOI: 10.1016/j.yhbeh.2015.12.001.

Houslay TM, Vullioud P, Zöttl M, Clutton-Brock TH. 2020. Benefits of cooperation in captive Damaraland mole-rats. Behavioral Ecology 31:711-718. DOI: 10.1093/beheco/araa015

Hurtado MJ, Fénéron R, Gouat P. 2013. Specialization in building tasks in the mound-building mouse, Mus spicilegus. Animal Behaviour 85:1153-1160. DOI: 10.1016/j.anbehav.2013.03.020.

Peer) reviewing PDF | (2020:05:49165:1:1:NEW 7 Aug 2020) 
453 Ingram DK. 2000. Age-related decline in physical activity: generalization to nonhumans.

454

455

456

457

458

459

460

461

462

463

464

465

466

467

468

469

470

471

472

473

474

Medicine and science in sports and exercise 32: 1623-1629. DOI: 10.1097/00005768200009000-00016.

Jacobs DS, Jarvis JUM. 1996. No Evidence for the Work-Conflict Hypothesis in the Eusocial Naked Mole-Rat (Heterocephalus glaber). Behavioral Ecology and Sociobiology 39:401-409.

Jarvis JUM. 1981. Eusociality in a mammal: cooperative breeding in naked mole-rat colonies. Science 212:571-573. DOI: 10.1126/science.7209555.

Jarvis JUM. 1991. Reproduction of Naked Mole-Rats. In: The Biology of the Naked Mole-Rat. Princeton, New Jersey: Princeton University Press, 384.

Jarvis JUM, O’Riain MJ, Bennett NC, Sherman PW. 1994. Mammalian eusociality: a family affair. Trends in Ecology and Evolution 9:47-51. DOI: 10.1016/0169-5347(94)90267-4.

Jarvis JUM, O’Riain MJ, McDaid E. 1991. Growth and factors affecting body size in naked mole-rats. In: Sherman PW, J. U. M. Jarvis, Alexander RD eds. The biology of the naked mole-rat. Princeton University Press, 358-383.

Jeanson R. 2019. Within-individual behavioural variability and division of labour in social insects. Journal of Experimental Biology 222. DOI: 10.1242/jeb.190868.

Jeanson R, Weidenmüller A. 2014. Interindividual variability in social insects - proximate causes and ultimate consequences. Biological Reviews 89:671-687. DOI: $10.1111 /$ brv.12074

Judd TM, Sherman PW. 1996. Naked mole-rats recruit colony mates to food sources. Animal Behaviour 52:957-969. DOI: 10.1006/anbe.1996.0244. 
475 Lacey EA, Alexander RD, Braude SH, Sherman PW, Jarvis JUM. 1991. An Ethogram for the 476 Naked Mole-Rat: Nonvocal Behaviors. In: The Biology of the Naked Mole-Rat. Princeton $477 \quad$ University Press, 209-242.

478 Lacey EA, Sherman PW. 1991. Social organization of naked mole-rat colonies: evidence for a 479 division of labor. In: The biology of the naked mole-rat. Princeton, NJ: Princeton $480 \quad$ University Press, 384-425.

481 Lacey EA, Sherman PW. 1997. Cooperative Breeding in Naked Mole-Rats: Implications for 482 Vertebrate and Invertebrate Sociality. In: Solomon NG, French JA eds. Cooperative 483 Breeding in Mammals. Cambridge: Cambridge University Press, 267-301. DOI:

484

485 486

487

488

489

490

491

492

493

494

495

496 10.1017/CBO9780511574634.011.

Langridge EA, Sendova-Franks AB, Franks NR. 2008. How experienced individuals contribute to an improvement in collective performance in ants. Behavioral Ecology and Sociobiology 62:447-456. DOI: 10.1007/s00265-007-0472-5.

Lever J, Krzywinski M, Altman N. 2016. Points of significance: model selection and overfitting. Nature Methods 13: 703-704. DOI: 10.1038/nmeth.3968.

Lüdecke D, Makowski D, Waggoner, P, Patil I. 2019. performance: assessment of regression models performance.

Marck A, Berthelot G, Foulonneau V, Marc A, Antero-Jacquemin J, Noirez P, Bronikowski AM, Morgan TJ, Garland Jr T, Carter PA, Hersen P. 2017. Age-related changes in locomotor performance reveal a similar pattern for Caenorhabditis elegans, Mus domesticus, Canis familiaris, Equus caballus, and Homo sapiens. Journals of Gerontology Series A: Biomedical Sciences and Medical Sciences 72: 455-463. DOI: 10.1093/gerona/glw136. 
497 Maynard Smith J, Szathmáry Eörs. 1999. The origins of life: From the birth of life to the origins $498 \quad$ of language. New York: Oxford University Press.

499 Mazerolle MJ, Linden D. 2019. AICcmodavg: Model Selection and Multimodel Inference Based $500 \quad$ on $(Q) A I C(c)$.

501 Medger K, Bennett NC, Ganswindt SB, Ganswindt A, Hart DW. 2019. Changes in prolactin, 502 cortisol and testosterone concentrations during queen succession in a colony of naked 503 mole-rats (Heterocephalus glaber): a case study. The Science of Nature 106:26. DOI:

504 10.1007/s00114-019-1621-1.

505

506

Min Y, Agresti A. 2002. Modeling Nonnegative Data with Clumping at Zero: A Survey. Journal of the Iranian Statistical Society 1:7-33.

507 508 509 510

511

512 513 514 515 516 517 518 519

Mongillo DL, Kosyachkova EA, Nguyen TM, Holmes MM. 2014. Differential effects of chronic fluoxetine on the behavior of dominant and subordinate naked mole-rats. Behavioural Brain Research 258:119-126. DOI: 10.1016/j.bbr.2013.10.023.

Mooney SJ, Filice DCS, Douglas NR, Holmes MM. 2015. Task specialization and task switching in eusocial mammals. Animal Behaviour 109:227-233. DOI: 10.1016/j.anbehav.2015.08.019.

Nakagawa S, Johnson PCD, Schielzeth $\mathrm{H}$. The coefficient of determination $\mathrm{R}^{2}$ and intra-class correlation coefficient from generalized linear mixed-effects models revisited and expanded. 2017. Journal of the Royal Society Interface 14: 20170213. DOI: 10.1098/rsif.2017.0213.

Nakagawa S, Schielzeth H. 2013. A general and simple method for obtaining R2 from generalized linear mixed-effects models. Methods in ecology and evolution 4: 133-142. DOI: 10.1111/j.2041-210x.2012.00261.x. 
520 Neumann C, Kulik L. 2020. EloRating: Animal Dominance Hierarchies by Elo Rating.

521 O'Riain MJ, Jarvis JUM. 1997. Colony member recognition and xenophobia in the naked mole522 rat. Animal Behaviour 53:487-498. DOI: 10.1006/anbe.1996.0299.

523 O'Riain MJ, Jarvis JUM. 1998. The dynamics of growth in naked mole-rats: the effects of litter 524 525 order and changes in social structure. Journal of Zoology 246:49-60. DOI:

526

527

528

529

530

531

532

533

534

535

536

537

538

539

540 10.1111/j.1469-7998.1998.tb00131.x.

Peroulakis ME, Goldman B, Forger NG. 2002. Perineal muscles and motoneurons are sexually monomorphic in the naked mole-rat (Heterocephalus glaber). Journal of neurobiology 51: 33-42. DOI: 10.1002/neu.10039.

R Core Team. 2014. R: A language and environment for statistical computing. $R$ Foundation for Statistical Computing. Vienna, Austria.

Reeve HK. 1992. Queen activation of lazy workers in colonies of the eusocial naked mole-rat. Nature 358:147-149. DOI: 10.1038/358147a0.

Robinson GE. 1992. Regulation of Division of Labor in Insect Societies. Annual Review of Entomology 37:637-665. DOI: 10.1146/annurev.en.37.010192.003225.

Sánchez-Tójar A, Schroeder J, Farine DR. 2018. A practical guide for inferring reliable dominance hierarchies and estimating their uncertainty. Journal of Animal Ecology 87:594-608. DOI: 10.1111/1365-2656.12776.

Santoro D, Hartley S, Lester PJ. 2019. Behaviourally specialized foragers are less efficient and live shorter lives than generalists in wasp colonies. Scientific Reports 9:1-10. DOI:

541 Schieffelin JS, Sherman PW. Tugging contests reveal feeding hierarchies in naked mole-rat 542 colonies. Animal behaviour 49: 537-541. DOI: 10.1006/anbe.1995.0073. 
543 Schielke CK, Begall S, Burda H. 2012. Reproductive state does not influence activity budgets of

544

545

546

547 Šklíba J, Lövy M, Burda H, Šumbera R. 2016. Variability of space-use patterns in a free living

548

549

550

551

552

553

554

555

556

557

558

559

560

561

562 Wickham H. 2016. ggplot2: Elegant Graphics for Data Analysis.

563 Wilson EO. 1987. Causes of Ecological Success: The Case of the Ants. Journal of Animal 564 eusocial Ansell's mole-rats, Fukomys anselli (Rodentia, Bathyergidae): A study of locomotor activity by means of RFID. Mammalian Biology 77: 1-5. DOI: 10.1016/j.mambio.2011.09.004. eusocial rodent, Ansell's mole-rat indicates age-based rather than caste polyethism. Scientific reports 6: 1-9. DOI: 10.1038/srep37497.

Stander PE. 1992. Cooperative hunting in lions: the role of the individual. Behavioral Ecology and Sociobiology 29. DOI: 10.1007/BF00170175.

Thorley J, Mendonça R, Vullioud P, Torrents-Ticó M, Zöttl M, Gaynor D, Clutton-Brock T. 2018. No task specialization among helpers in Damaraland mole-rats. Animal Behaviour 143:9-24. DOI: 10.1016/j.anbehav.2018.07.004.

Van Daele PAAG, Desmet N, Šumbera R, Adriaens D. 2019. Work behaviour and biting performance in the cooperative breeding Micklem's mole-rat Fukomys micklemi (Bathyergidae, Rodentia). Mammalian Biology 95:69-76. DOI:

10.1016/j.mambio.2019.02.002.

de Vries H, Stevens JMG, Vervaecke H. 2006. Measuring and testing the steepness of dominance hierarchies. Animal Behaviour 71:585-592. DOI: 10.1016/j.anbehav.2005.05.015. Ecology 56:1-9. DOI: 10.2307/4795. 
565 Zöttl M, Vullioud P, Mendonça R, Torrents Ticó M, Gaynor D, Mitchell A, Clutton-Brock T.

566 2016. Differences in cooperative behavior among Damaraland mole rats are

567 consequences of an age-related polyethism. Proceedings of the National Academy of

$568 \quad$ Sciences 113:10382-10387. DOI: 10.1073/pnas.1607885113.

569 


\section{Figure 1 (on next page)}

Presence of working behaviour by age (A), weight (B) and rank (C) of the individual being observed.

The plots are fitted with locally weighted (loess) regression lines that display localised trends in the data. These are different from the models described below which include colony and individual as random effects. The size of the points reflects the number of data points at each location (geom_count, ggplot2). Rank is scaled to account for group size and the most dominant individuals have ranks closer to zero. Age and weight-cubed were associated with the probability that an individual was observed working; younger and mid-sized individuals worked most frequently. 
Figure 2 (on next page)

Duration of working behaviour by age (A), weight (B) and rank (C) of the individual being observed.

The plots are fitted with locally weighted (loess) regression lines that display localised trends in the data. These are different from the models described below which include colony and individual as random effects. Rank is scaled to account for group size and the most dominant individuals have ranks closer to zero. Rank-squared was associated with the duration an individual was observed working; more dominant animals worked for shorter periods of time. 


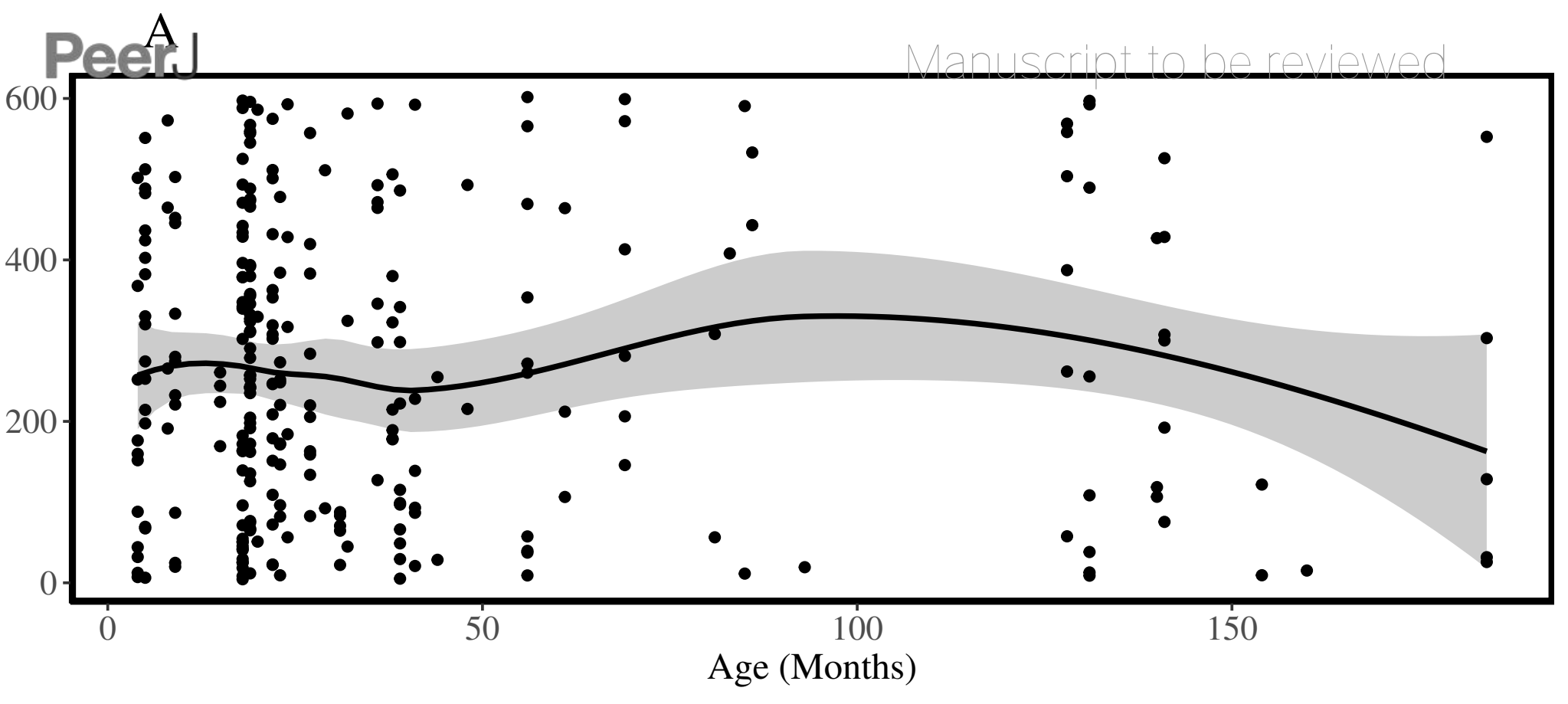

B
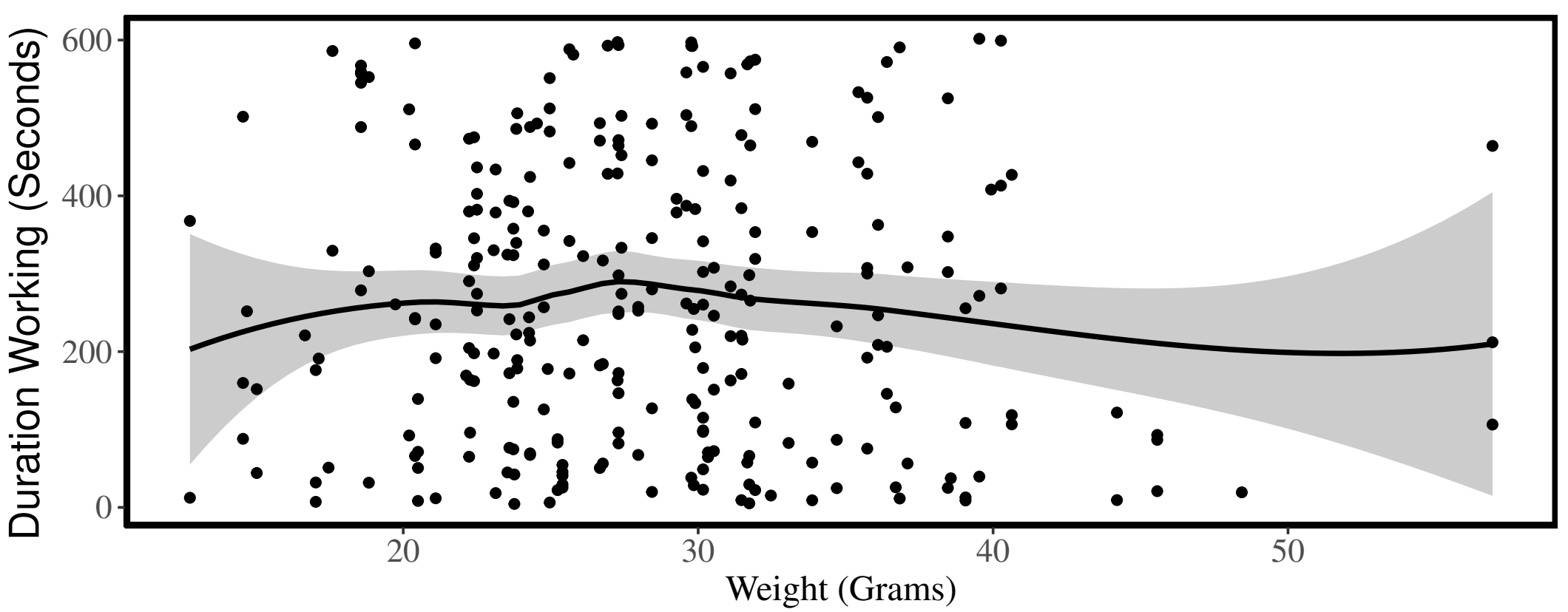

C

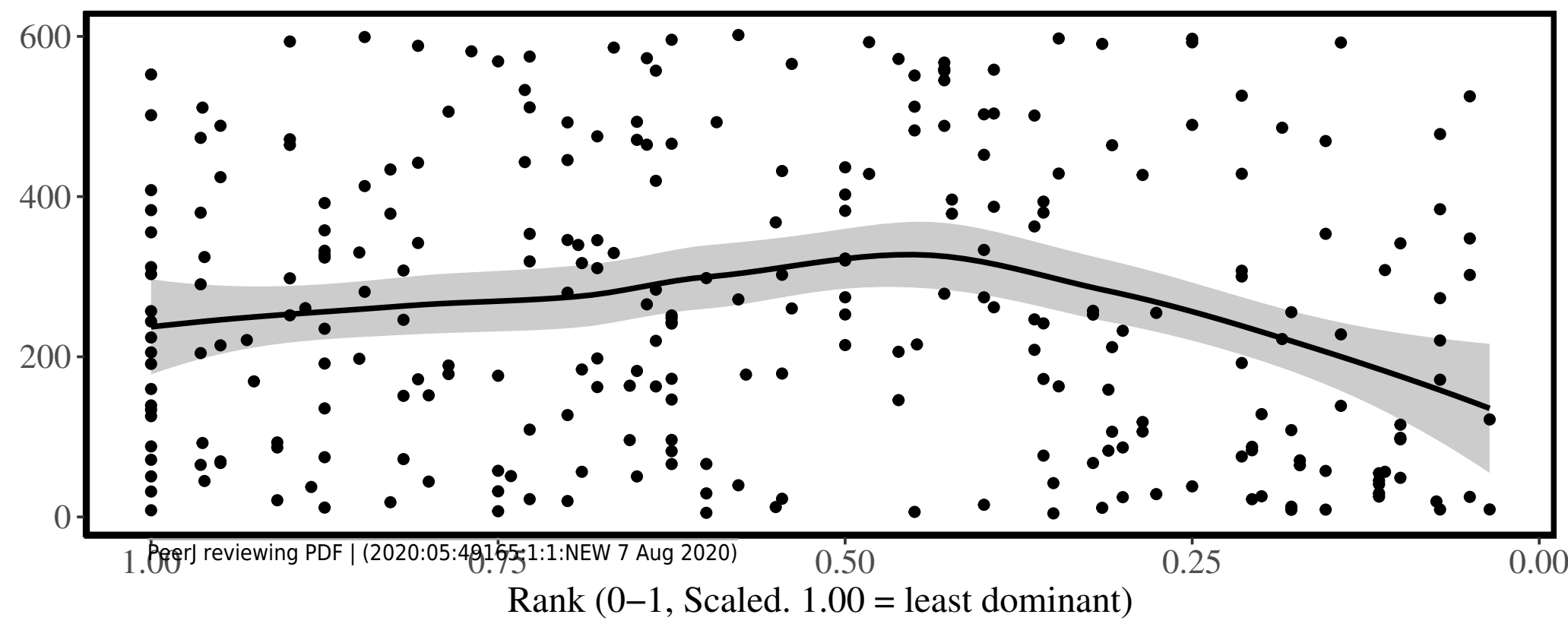




\section{Figure 3}

Figure 3. Duration of working behaviour per observation period for each colony.

Medians are indicated by thick black horizontal lines, the upper and lower quartiles are represented by the upper and lower edges of the boxes, and the whiskers extend to 1.5 the interquartile ranges. Data points outside the whiskers are plotted as points. 
Figure 3. Duration of working behaviour per observation period for each colony.

Medians are indicated by thick black horizontal lines, the upper and lower quartiles are represented by the upper and lower edges of the boxes, and the whiskers extend to 1.5 the interquartile ranges. Data points outside the whiskers are plotted as points.

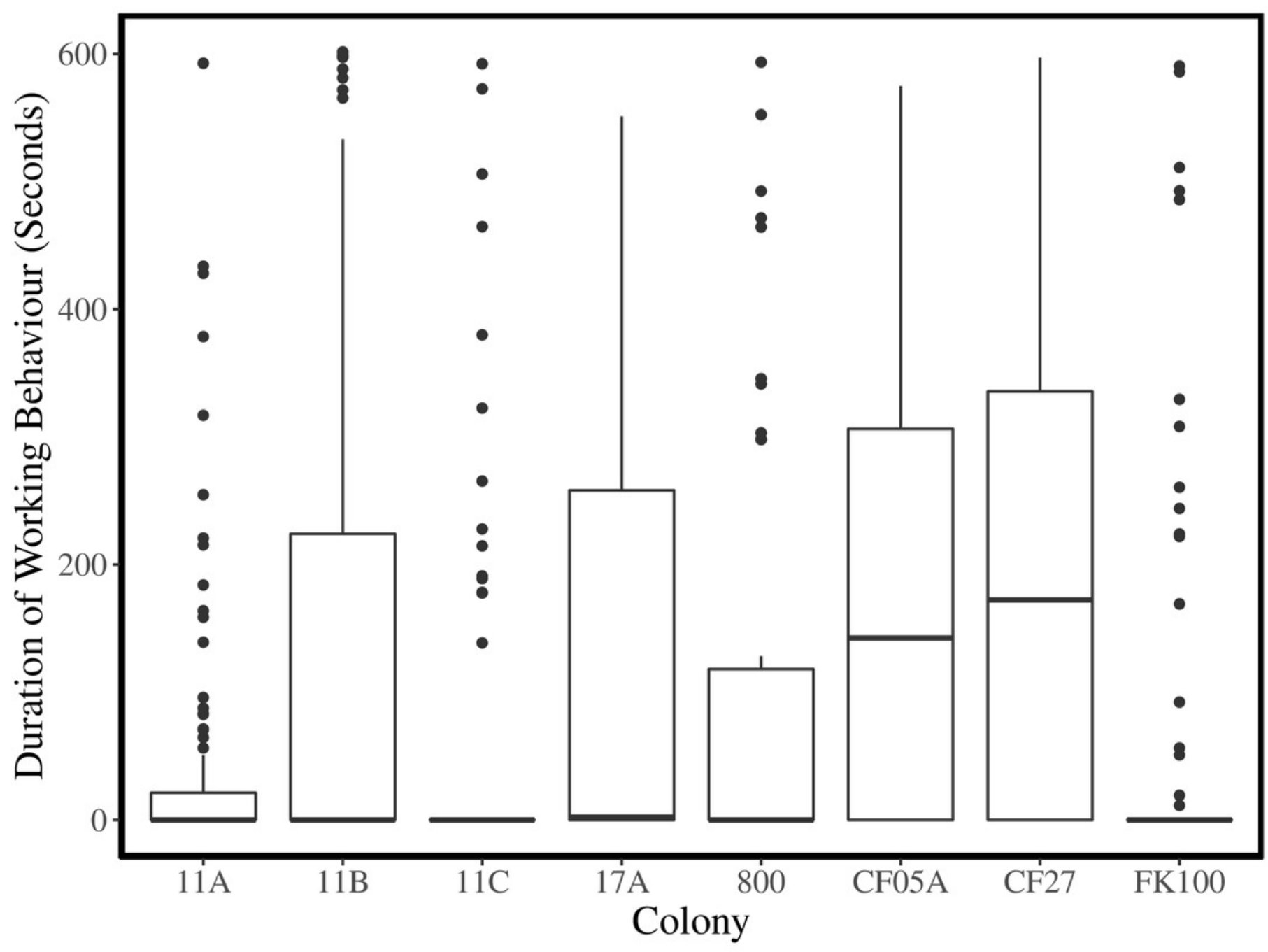


Figure 4 (on next page)

Presence of working behaviour by age (A), weight (B) and rank (C) of the individual being observed for each colony.

The lines are generalised linear regressions with a binary response variable (whether work was observed during the observation period) using ggplot2's stat_smooth function (Wickham 2016). The size of the points reflects the number of data points at each location (geom_count, ggplot2). 
Peêr J
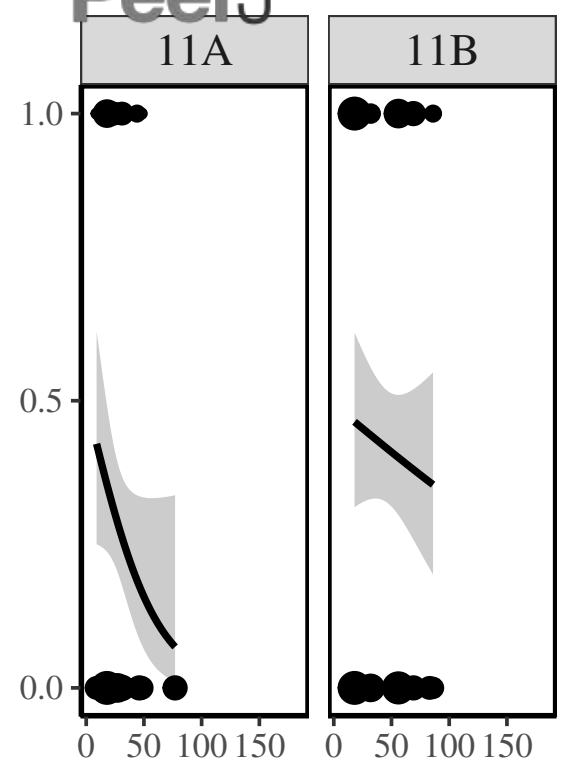

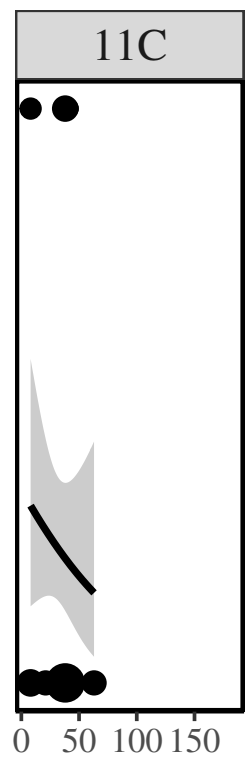

Ma

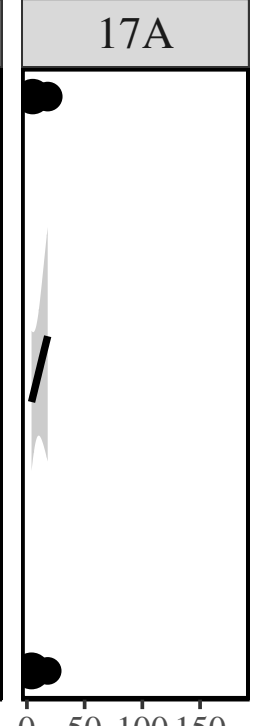

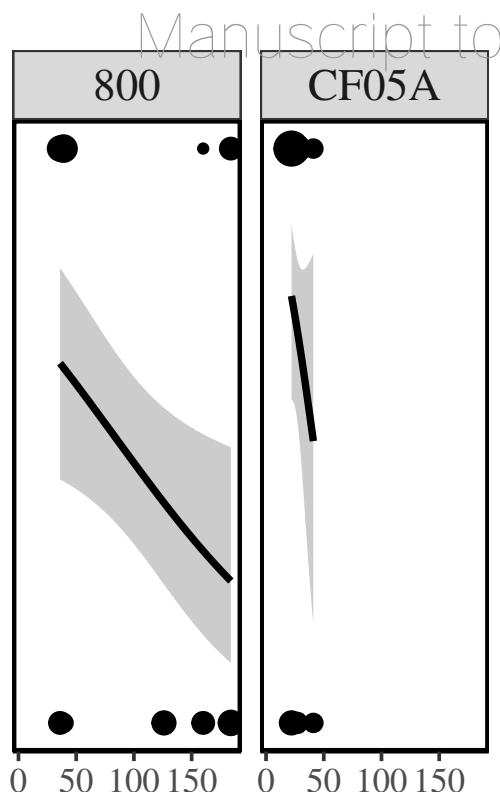

Age (Months)

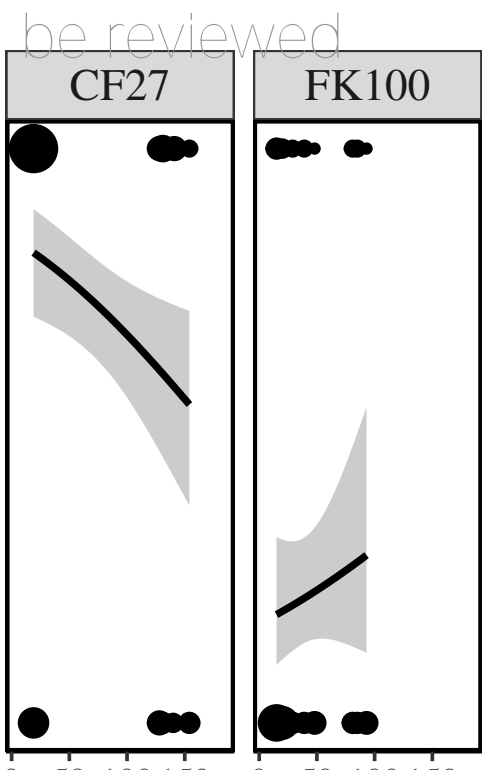

B
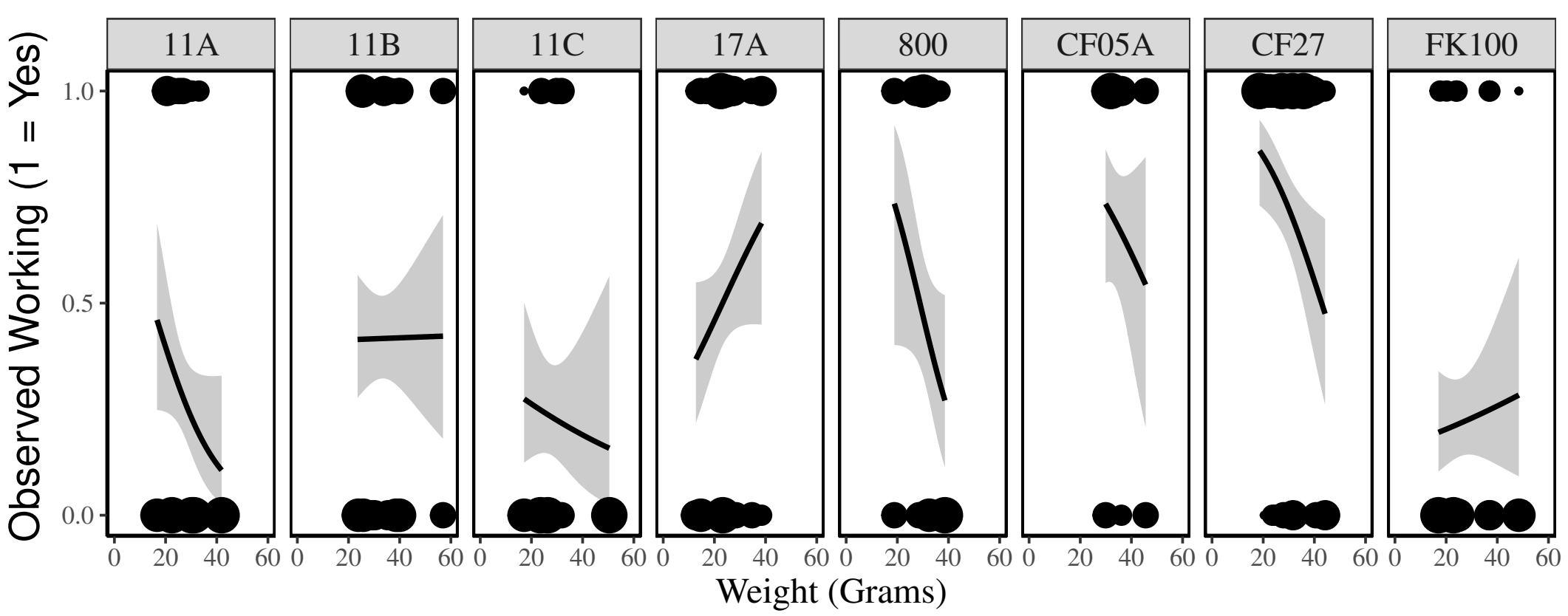

C
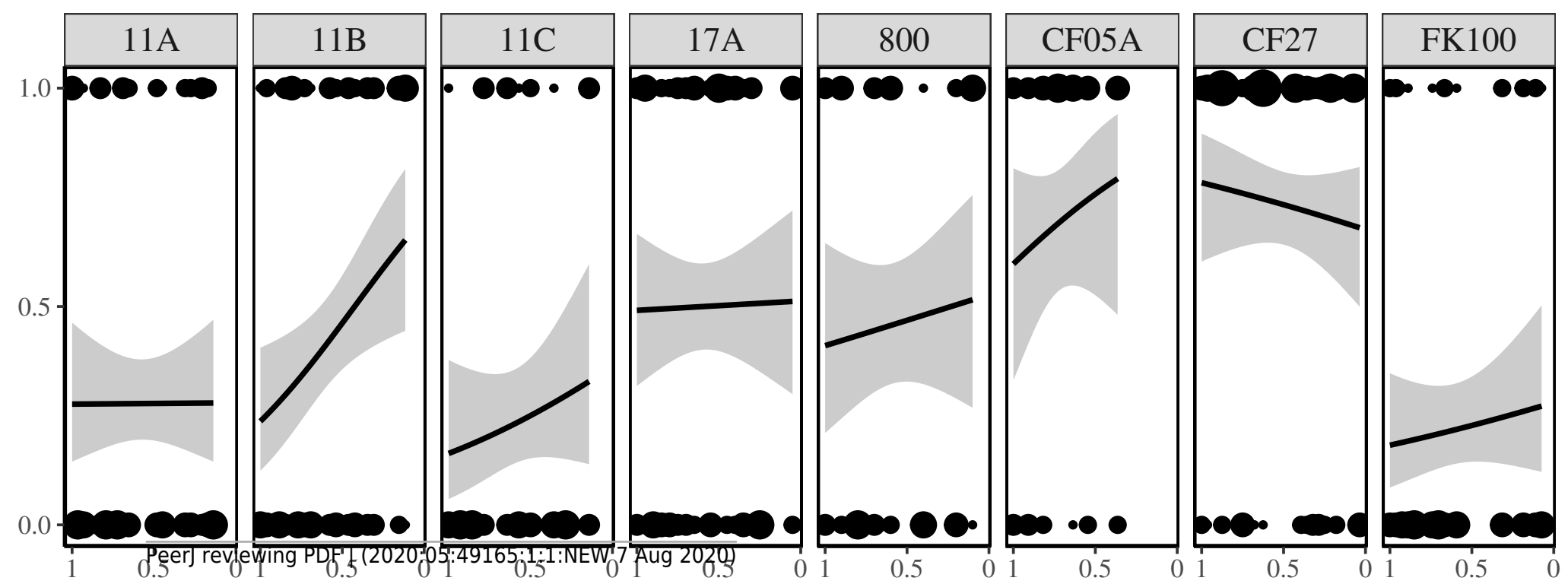

Rank $(0-1$, Scaled. $1.00=$ least dominant $)$ 
Figure $\mathbf{5}$ (on next page)

Figure $5 \mathrm{~A}-\mathrm{C}$. Duration of working behaviour by age (A), weight (B) and rank $(C)$ of the individual being observed for each colony.

The lines are general linear regressions with duration of working behaviour as the response variable using ggplot2's stat_smooth function (Wickham 2016). 
PeAj

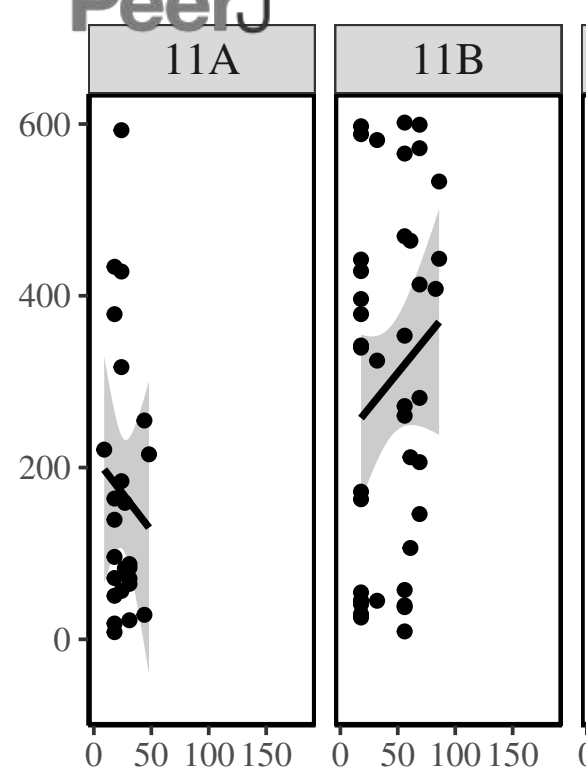

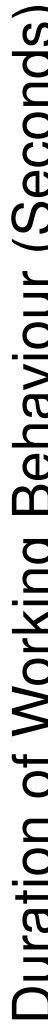

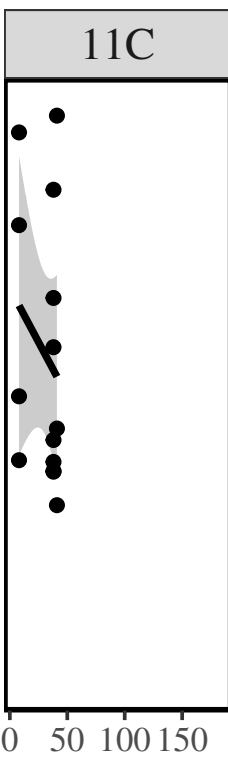

.

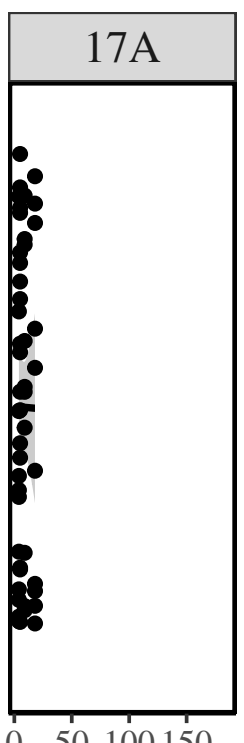

Age (Months)
Manuscript to be reviewed
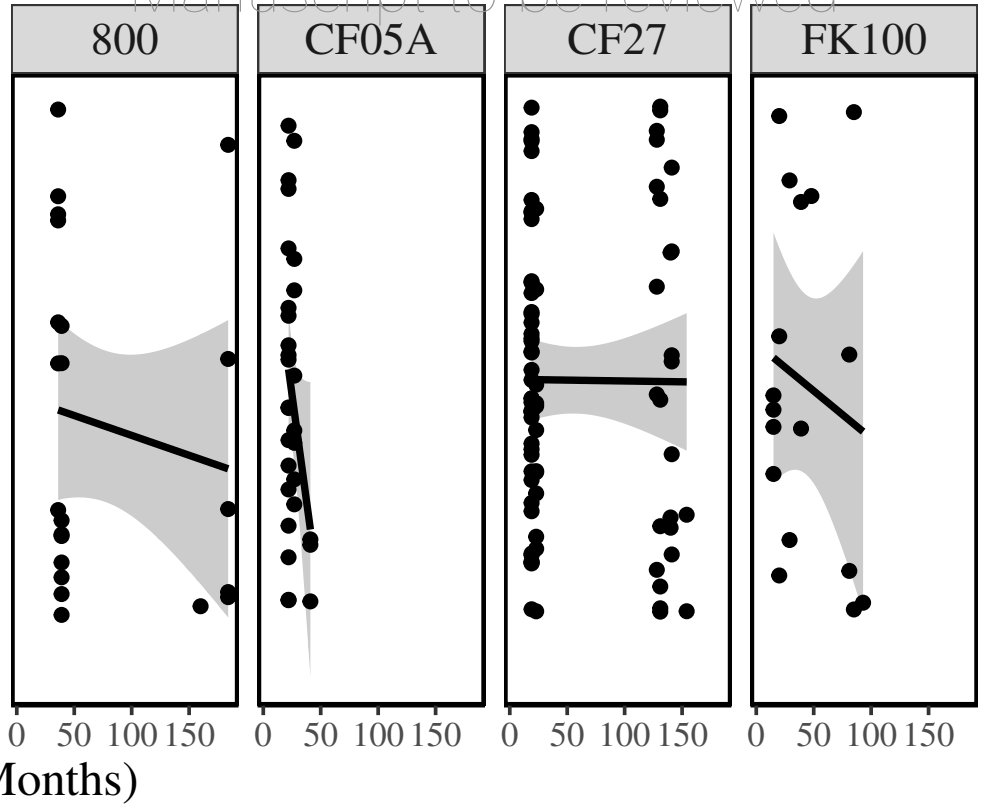

50100150
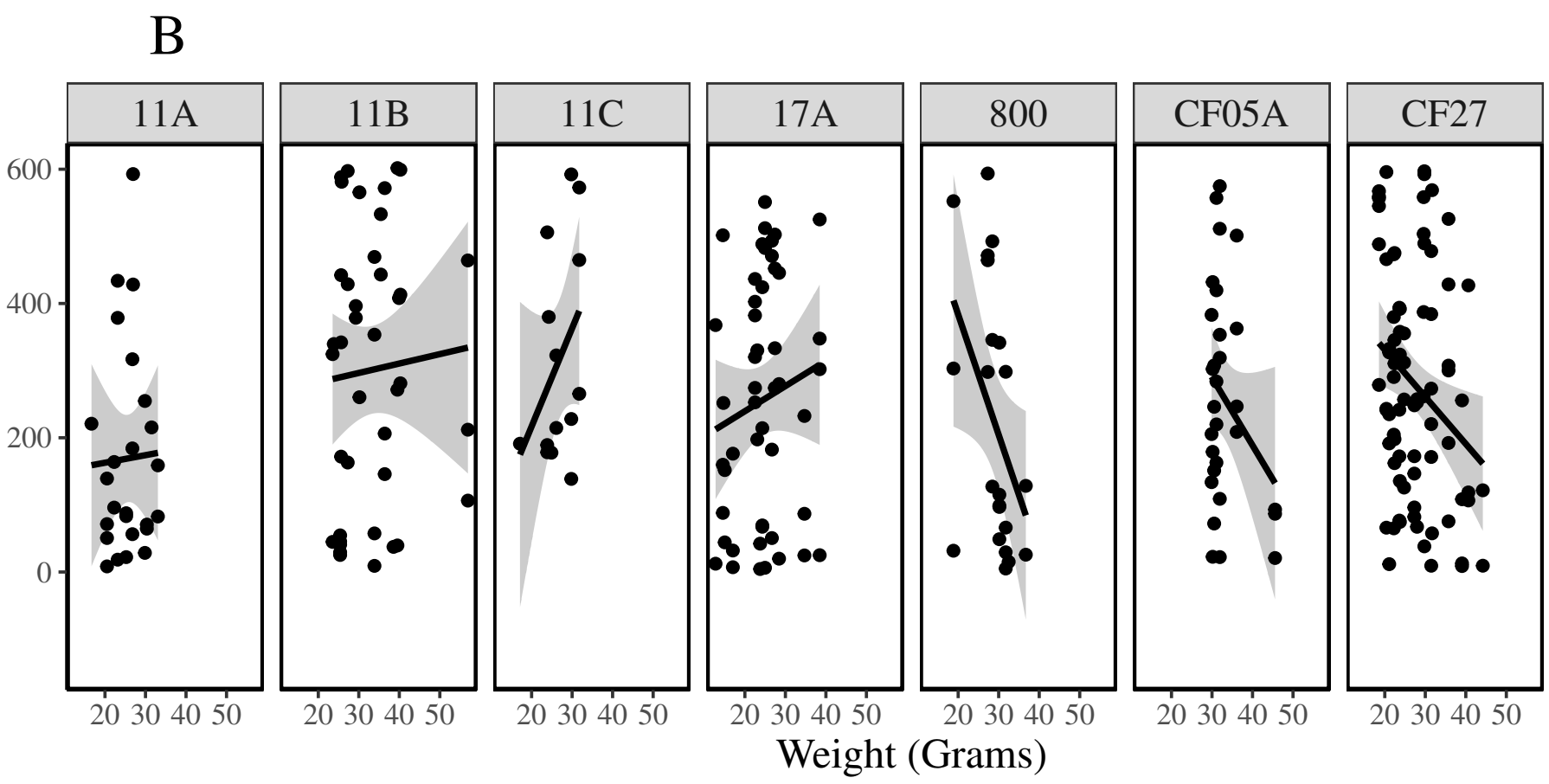

FK100

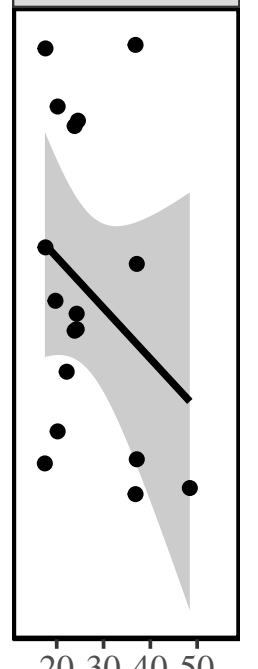

20304050
C

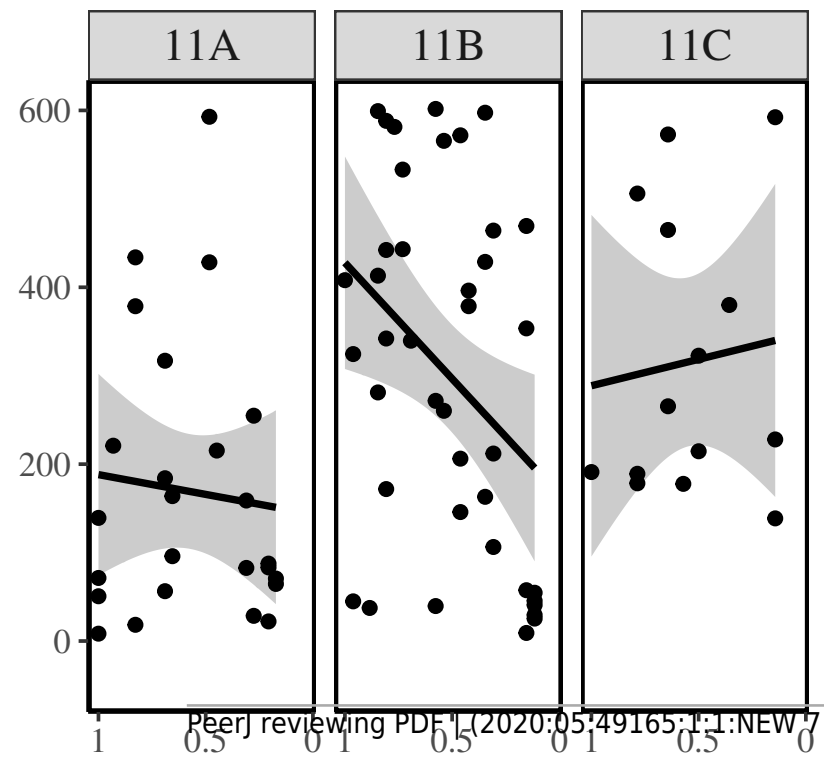

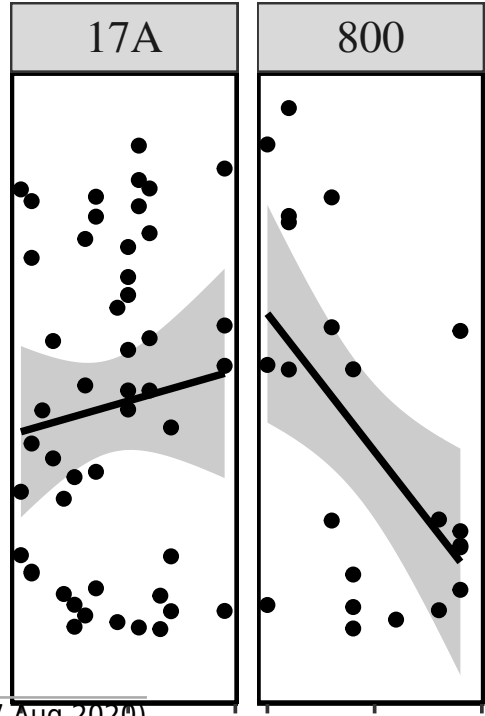

01

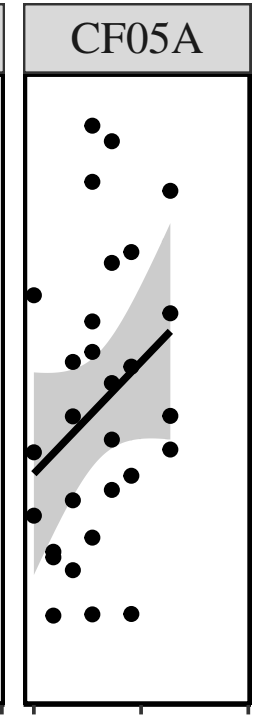

01

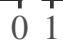

0.5

Rank $(0-1$, Scaled. $1.00=$ least dominant $)$

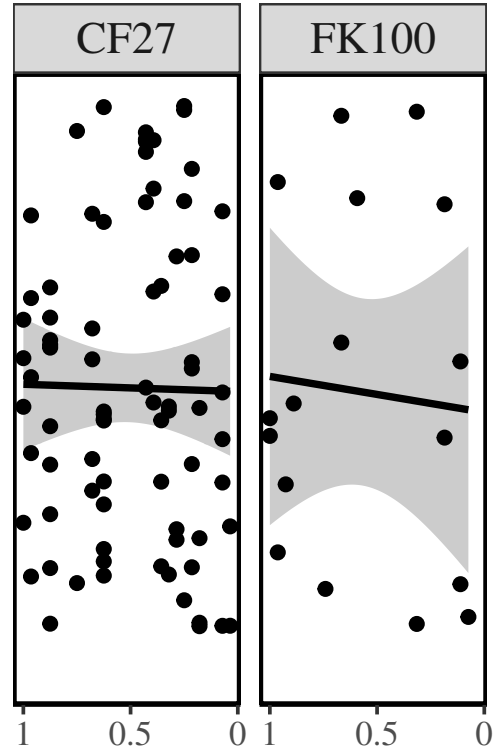




\section{Table $\mathbf{1}$ (on next page)}

Table 1. Summary statistics of animals used in the study 
1 Table 1. Summary statistics of animals used in the study.

\begin{tabular}{|c|c|c|c|c|c|c|c|}
\hline Colony & $\begin{array}{c}\text { Total } \\
\text { Number of } \\
\text { Individuals } \\
\text { (at start of } \\
\text { observation } \\
\text { period) }\end{array}$ & $\begin{array}{c}\text { Number of } \\
\text { Individuals } \\
\text { Observed }\end{array}$ & $\begin{array}{c}\text { Number } \\
\text { of } \\
\text { Females } \\
\text { Observed }\end{array}$ & $\begin{array}{c}\text { Proportion } \\
\text { Female }\end{array}$ & $\begin{array}{c}\text { Mean (min, } \\
\text { max) Age } \\
\text { (months) }\end{array}$ & $\begin{array}{c}\text { Mean (min, } \\
\text { max) Weight } \\
\text { (grams) }\end{array}$ & $\begin{array}{c}\text { Mean (SD) } \\
\text { Behaviour } \\
\text { Recorded } \\
\text { (seconds per } \\
\text { observation) }\end{array}$ \\
\hline $11 \mathrm{~A}$ & 28 & 15 & 6 & 0.40 & $30.7(9,77)$ & $27.5(16.7,41.9)$ & $47(110.5)$ \\
\hline $11 \mathrm{~B}$ & 24 & 16 & 7 & 0.43 & $46.6(18,86)$ & $33.2(23.5,56.9)$ & $125.5(197.5)$ \\
\hline $11 \mathrm{C}$ & 16 & 10 & 8 & 0.80 & $33.1(8,63)$ & $27.8(17.1,50.4)$ & $73.7(153.9)$ \\
\hline $17 \mathrm{~A}$ & 23 & 16 & 10 & 0.63 & $7.9(4,18)$ & $23.2(12.8,38.5)$ & $128.1(180.7)$ \\
\hline 800 & 10 & 8 & 7 & 0.88 & $100.5(36,184)$ & $30.5(18.8,38.6)$ & $103.1(173.1)$ \\
\hline CF05A & 10 & 7 & 2 & 0.29 & $26.1(22,41)$ & $33.6(29.9,45.6)$ & $178.3(180.9)$ \\
\hline CF27 & 26 & 18 & 13 & 0.72 & $65(19,154)$ & $28.6(18.6,44.2)$ & $203.6(192.3)$ \\
\hline FK100 & 43 & 13 & 5 & 0.38 & $38.1(15,93)$ & $25.5(17,48.4)$ & $59.7(145.4)$ \\
\hline Total & 180 & 103 & 58 & 0.56 & $41.9(4,184)$ & $56.9)$ & $116.6(177.5)$ \\
\hline
\end{tabular}




\section{Table 2 (on next page)}

Table 2. The average duration of working behaviour and percentage of time observed working per 600-second observation period for females, males and both sexes combined. 
1 Table 2. The average duration of working behaviour and percentage of time observed

2 working per 600-second observation period for females, males and both sexes combined.

\begin{tabular}{|c|c|c|}
\hline Sex & $\begin{array}{c}\text { Average time observed in } \\
\text { working behaviour (SD) }\end{array}$ & $\begin{array}{c}\text { Percentage of time observed } \\
\text { working }\end{array}$ \\
\hline Female & $124.8(179.2)$ & 20.8 \\
\hline Male & $106.1(174.9)$ & 17.7 \\
\hline Combined & $116.6(177.5)$ & 19.4 \\
\hline
\end{tabular}

3 


\section{Table 3 (on next page)}

Results of logistic mixed-effects models predicting whether working behaviour was observed or not.

The format of the models was: Presence or absence of working behaviour Colony|Individual

+ Fixed Effect Where Presence of working behaviour is whether working behaviour was observed during the ten-minute observation period, Colony and Individual are random effects with Individual nested within Colony, and Fixed Effect was omitted in the null model and one of sex, age (months), weight (grams) and rank (0-1, scaled) was included in the corresponding models. The scripts used to run the models are available in Supplementary

File 3. Relative likelihoods are calculated as $\exp (-(1 / 2) \Delta A I C C)$. Nakagawa's $R^{2}$ estimates the variance explained by the fixed effects (marginal variance) and both fixed and random effects (conditional variance) (Nakagawa \& Schielzeth, 2013). 
1

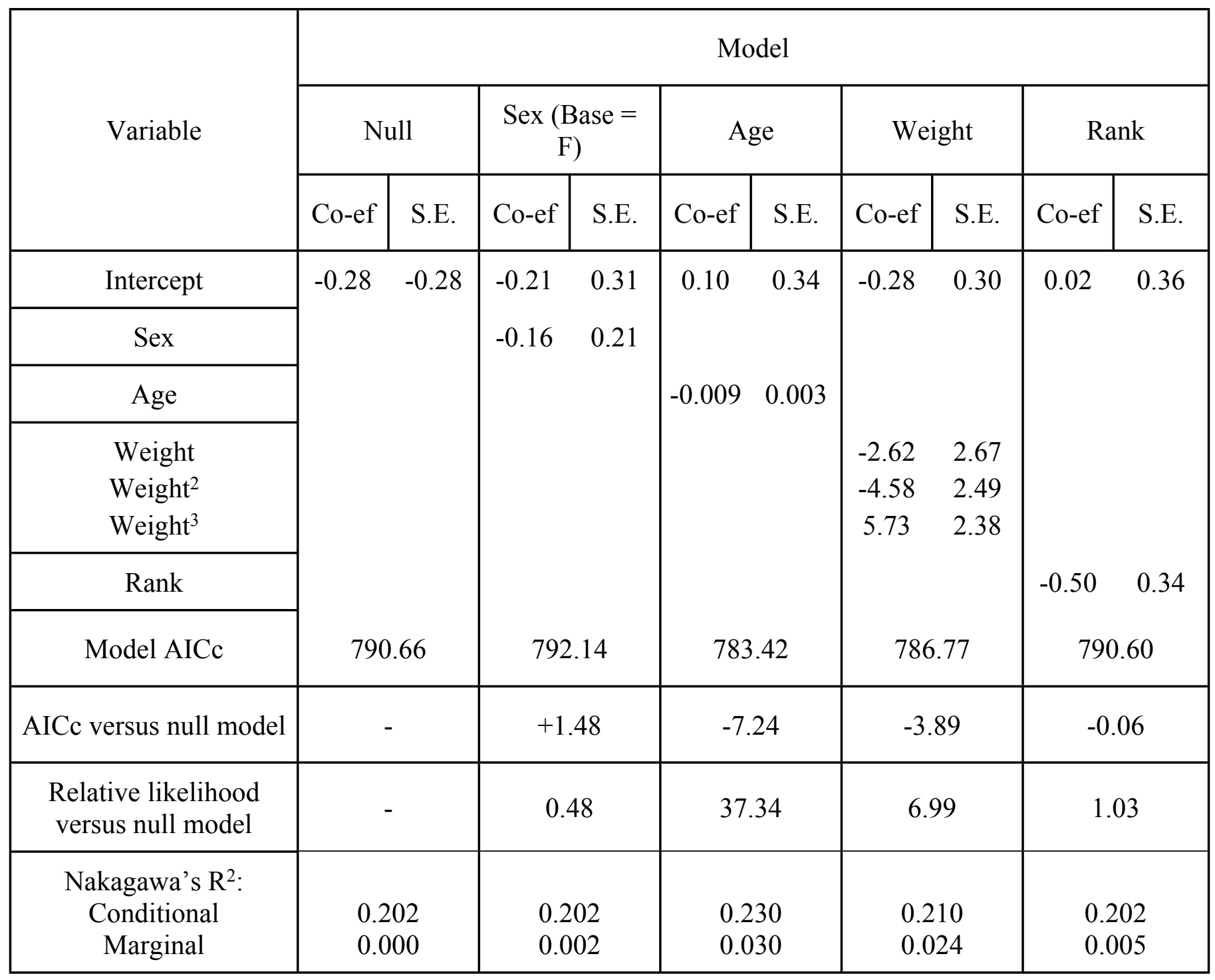

2 


\section{Table 4 (on next page)}

Results of generalised linear mixed-effects models predicting the duration of observed working behaviour.

The format of the models was: Duration of working behaviour $\sim$ Colony|Individual + Fixed Effect Where Duration of working behaviour is the duration of working behaviour observed during the ten-minute observation period, Colony and Individual are random effects with Individual nested within Colony, and Fixed Effect was omitted in the null model and one of sex, age (months), weight (grams) and rank (0-1, scaled) was included in the corresponding models. The scripts used to run the models are available in Supplementary File 3. Relative likelihoods are calculated with $\exp (-(1 / 2) \Delta A I C C)$. Nakagawa's $\mathrm{R}^{2}$ estimates the variance explained by the fixed effects (marginal variance) and both fixed and random effects (conditional variance) (Nakagawa \& Schielzeth, 2013). 
1

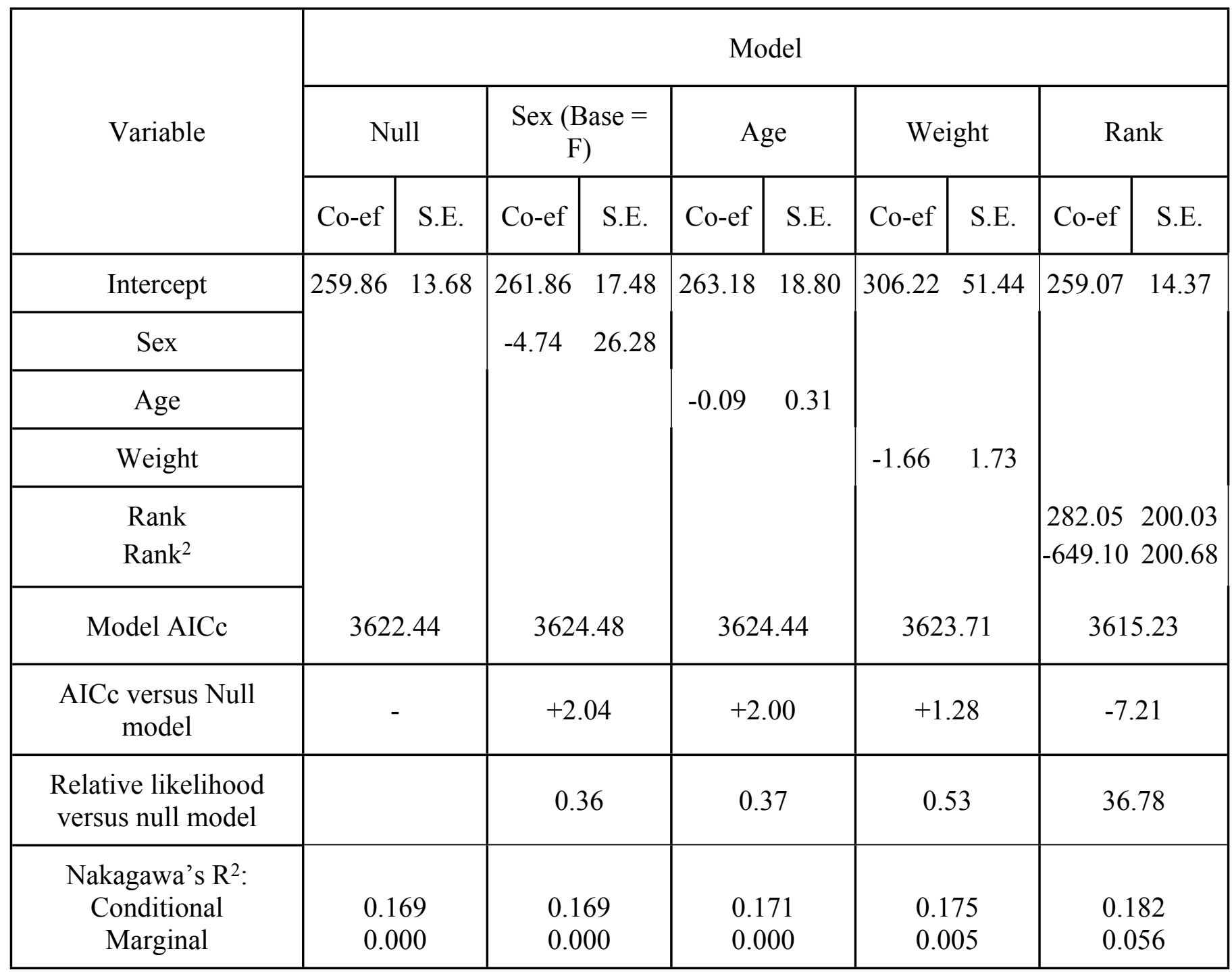

